\title{
DETERMINANTES E CONDICIONANTES SOCIAIS: FORMAS DE UTILIZAÇÃO NOS PLANOS NACIONAL E ESTADUAIS DE SAÚDE
}

\author{
SOCIAL CONDITIONANTS AND DETERMINANTS: FORMS OF \\ USE IN THE NATIONAL AND STATE HEALTH PLANS
}

DETERMINANTES Y CONDICIONANTES SOCIALES: FORMAS DE

UTILIZACIÓN EN LOS PLANES NACIONAL Y ESTADUAL DE SALUD

\author{
André Luis Alves de Quevedo ${ }^{1}$ \\ Carmen Luisa Teixeira Bagatini ${ }^{2}$ \\ Maria Isabel Barros Bellini ${ }^{3}$ \\ Rebel Zambrano Machado ${ }^{4}$ \\ Camila Guaranha ${ }^{5}$
}

Resumo O objetivo da pesquisa foi descrever como o tema dos determinantes e condicionantes de saúde foi contemplado no plano nacional e nos planos estaduais de saúde elaborados para o período de 2012 a 2015. Tratou-se de pesquisa qualitativa, do tipo análise documental, com foco no tema dos determinantes e condicionantes de saúde. Os referidos documentos foram buscados na página do Ministério da Saúde e nas das secretarias estaduais de saúde das 27 unidades federativas brasileiras. A busca também foi realizada no Sistema de Apoio à Construção do Relatório de Gestão, no website Google e com o envio de e-mails. Observou-se que o tema dos determinantes e condicionantes de saúde está pouco presente nos planos de saúde brasileiros. A maioria dos documentos utilizou o referencial do planejamento em saúde em perspectiva normativa, conforme proposto em documentos técnicos do Ministério da Saúde. A discussão sobre modelos de determinantes e condicionantes de saúde não estava presente nos documentos analisados, e houve uma descrição mista do tema por meio de dados desagregados e índices compostos. O estudo indicou a necessidade de maior atenção e revisão dos modelos de determinantes e condicionantes de saúde empregados na construção desses documentos técnicos.

Palavras-chave determinantes sociais da saúde; planos governamentais de saúde; planejamento em saúde; Sistema Único de Saúde.
Abstract The study aimed to describe how the health conditionants and determinants topic was covered in the national and state health plans drafted for the period ranging from 2012 to 2015 . It was a qualitative, documentary analysis-type study focusing on the health conditionants and determinants topic. These documents were queried on the websites of the Ministry of Health and of the state health departments of the 27 Brazilian states. Research was also carried out in the Management Report Construction Support System, on Google, and by e-mail. It was noted that the health conditionants and determinants issue is not present in Brazilian health plans. Most of the documents used the health planning framework in a normative perspective, as proposed in the Ministry of Health's technical documents. The discussion on health conditionant and determinant models was not featured among the documents that were analyzed, and there was a mixed description of the topic through disaggregated data and composite indices. The study indicated the need for more attention and review of the health conditionant and determinant models used to prepare these technical documents.

Keywords social determinants of health; government health plans; health planning; Unified Health System. 


\section{Introdução}

O plano nacional e os planos estaduais de saúde são documentos oficiais que direcionam as diretrizes da política de saúde durante um período de quatro anos em determinado território. Demonstram a intenção e o compromisso dos gestores com as questões relativas à saúde, constituindo-se, portanto, em instrumento do planejamento do governo.

Na portaria n. 3.332, de 28 de dezembro de 2006, que trazia à discussão a institucionalização e o fortalecimento do planejamento no Sistema Único de Saúde (SUS), esse tema aparecia como eixo estruturante da análise situacional e na formulação dos objetivos, diretrizes e metas, primando pela intersetorialidade (Brasil, 2006). No entanto, foi revogada pela portaria n. 2.135, de 25 de setembro de 2013, que a atualizou (Brasil, 2013).

Atualmente, a portaria n. 2.135/2013 estabelece as diretrizes para o planejamento no âmbito do SUS e reitera a necessidade de compatibilização entre os instrumentos de planejamento da saúde (plano de saúde e respectivas programações anuais, relatórios de gestão quadrimestrais e anuais) e os instrumentos de planejamento e orçamento de governo, como o Plano Plurianual (PPA), a Lei de Diretrizes Orçamentárias (LDO) e a Lei Orçamentária Anual (LOA), em cada esfera de gestão (Brasil, 2013). Segundo essa portaria, os planos de saúde devem considerar: a análise situacional, a definição das diretrizes, os objetivos, metas e indicadores e o processo de monitoramento e avaliação.

Na portaria n. 2.135/2013, não está explícito o tema dos determinantes e condicionantes, fundamentais para análise da situação de saúde. A referida portaria aponta apenas que a análise situacional deve contemplar os temas do Mapa da Saúde proposto pelo Ministério da Saúde. Nesse mapa, nas condições sociossanitárias, consta o tema determinantes sociais de saúde.

A Organização Mundial da Saúde (OMS) criou em 2005 a Comissão sobre Determinantes Sociais da Saúde; e em 2006 foi criada a Comissão Nacional sobre Determinantes Sociais da Saúde (CNDSS) no Brasil (Buss e Pellegrini Filho, 2006).

Como um dos resultados do trabalho da comissão foi produzido o documento intitulado "Rumo a um modelo conceitual para análise e ação sobre os determinantes sociais de saúde", no qual são apresentados alguns modelos sobre determinantes e condicionantes de saúde utilizados no mundo: Dahlgren e Whitehead - influências em camadas; Diderichsen e colaboradores - estratificação social e produção de doenças; Mackenbach e colaboradores - seleção e causa; Brunner, Marmot e Wilkinson - múltiplas influências no decorrer da vida, a proposta de modelo conceitual para a CNDSS e o Modelo de Stronks, proposto no contexto do Programa Holandês Nacional de Pesquisa sobre as desigualdades na saúde (Comissão Nacional sobre Determinantes Sociais da Saúde, 2005).

Entende-se que a escolha de um modelo para tratar de condicionantes e determinantes de saúde é a primeira etapa a ser realizada para a construção 
da análise da situação de saúde de um determinado território. A partir do modelo escolhido, faz-se necessário identificar as dimensões apresentadas no modelo adotado; e num terceiro momento, deve-se selecionar os indicadores ou índices que se relacionam com essas dimensões.

Nesse sentido, o objetivo da pesquisa apresentada neste artigo foi descrever como o tema dos determinantes e condicionantes de saúde foi contemplado no plano nacional e nos planos estaduais de saúde referentes ao período de 2012 a 2015.

\section{Percurso metodológico: investigando os determinantes e condicionantes no Plano Nacional e planos estaduais de saúde}

Tratou-se de uma pesquisa qualitativa do tipo análise documental. Os documentos analisados foram o plano nacional e os planos estaduais de saúde de 24 das 27 unidades federativas brasileiras para o período de 2012 a 2015, referentes ao tema dos determinantes e condicionantes de saúde. Os planos foram buscados na página do Ministério da Saúde e das secretarias estaduais de saúde, nas áreas de planejamento, instrumentos de gestão e transparência pública; e quando não localizados, recorreu-se, consecutivamente, às páginas dos conselhos estaduais de saúde, ao Sistema de Apoio à Construção do Relatório de Gestão (SARGSUS) ou ao website Google. Em última instância, foram enviados e-mails às secretarias estaduais de saúde em busca de recuperar os documentos não encontrados.

Para a análise documental qualitativa, construiu-se uma tabela de registro dos recortes nos documentos analisados que indicavam o tema em estudo, e foi realizada uma análise preliminar dos resultados selecionados. Em seguida, sistematizaram-se as observações com a identificação dos pré-temas que orientaram uma segunda leitura dos documentos e da tabela de análise, visando à identificação de aspectos relevantes e de sua descrição e análise crítica, no sentido de apontar as diferentes perspectivas adotadas, seus pontos de confluência e de conflito. Assim, os dados referentes às propostas sobre determinantes e condicionantes de saúde expressos no plano nacional e nos planos estaduais de saúde para o período estudado foram captados por meio da identificação de temas (Bardin, 2010; Minayo, 2014). Observaram-se, ainda, a articulação com conselhos de saúde e o seguimento das recomendações de conferências de saúde das respectivas instâncias.

\section{O 'estado da arte' do tema analisado: uma abordagem da federação e regiões geográficas brasileiras}

O plano nacional e os planos estaduais de saúde de vinte unidades federativas estavam disponíveis nas páginas oficiais. Um plano estadual de saúde foi identificado no sistema SARGSUS e um no website Google; dois documentos foram recuperados por meio de e-mails recebidos das secretarias 
estaduais de saúde, e três planos estaduais de saúde da região Norte não foram recuperados.

O Quadro 1 apresenta os conteúdos utilizados nos planos analisados para abordar o tema determinantes e condicionantes de saúde.

Quadro 1

\begin{tabular}{|c|c|c|}
\hline Unidade federativa & Conteúdos abordados & Páginas \\
\hline União (Brasil) & $\begin{array}{l}\text { Aspectos socioeconômicos, condições de vida, trabalho e ambiente, hábitos e estilos de vida, complexo } \\
\text { produtivo e de ciência, tecnologia e inovação em saúde }\end{array}$ & 28 a 37 \\
\hline \multicolumn{3}{|l|}{ Região Centro-Oeste } \\
\hline Distrito Federal (DF) & Não há descrição clara do tema no documento analisado & - \\
\hline Goiás (GO) & $\begin{array}{l}\text { Aspectos socioeconômicos, taxa de analfabetismo, condições de vida, trabalho e ambiente, complexo } \\
\text { produtivo e de ciência, tecnologia e inovação em saúde }\end{array}$ & 38 a 40 \\
\hline Mato Grosso (MT) & $\begin{array}{l}\text { Demografia, expectativa de vida ao nascer, índice de envelhecimento, razão de dependência, urbanização, } \\
\text { Índice de Desenvolvimento Humano (IDH) }\end{array}$ & 22 a 29 \\
\hline Mato Grosso do Sul (MS) & $\begin{array}{l}\text { Produto interno bruto (PIB) per capita, IDH, taxa de urbanização e densidade demográfica, população em } \\
\text { idade ativa e economicamente ativa (2006-2009), além de outros indicadores macroeconômicos }\end{array}$ & 15 a 21 \\
\hline \multicolumn{3}{|l|}{ Região Nordeste } \\
\hline Alagoas (AL) & $\begin{array}{l}\text { Grau de urbanização, razão de dependência, taxa de analfabetismo, PIB per capita, proporção de pobres, taxa } \\
\text { de desemprego, taxa de trabalho infantil, taxa de fecundidade total, taxa bruta padronizada de natalidade }\end{array}$ & 26 a 32 \\
\hline Bahia (BA) & Não há descrição clara do tema no documento analisado & - \\
\hline Ceará (CE) & $\begin{array}{l}\text { Aspectos econômicos, dados demográficos, indicadores sociais, renda, saúde, educação, indicador } \\
\text { de desenvolvimento: habitação, acesso a bens de consumo, saneamento e coleta de lixo, atividades } \\
\text { econômicas e outros indicadores econômicos }\end{array}$ & 33 a 45 \\
\hline Maranhão (MA) & Demografia, IDH, educação, trabalho e renda, saneamento básico, vigilância sanitária & $\begin{array}{c}172 \mathrm{a} \\
183\end{array}$ \\
\hline Paraíba (PB) & Não há descrição clara do tema no documento analisado & - \\
\hline Pernambuco (PE) & Não há descrição clara do tema no documento analisado & - \\
\hline Piauí (PI) & Não há descrição clara do tema no documento analisado & - \\
\hline $\begin{array}{l}\text { Rio Grande do Norte } \\
\text { (RN) }\end{array}$ & $\begin{array}{l}\text { IDH, taxa de urbanização, indicadores de saneamento básico, Indicador Social de Desenvolvimento dos } \\
\text { Municípios (ISDM: abrange habitação, renda, trabalho, saúde e educação, e ainda } 28 \text { variantes desses } \\
\text { componentes), renda familiar per capita abaixo da linha da pobreza, índice de mortalidade infantil, índice } \\
\text { de doenças evitáveis em crianças menores de cinco anos, Índice de Desenvolvimento da Educação Básica } \\
\text { (Ideb), proporção da população em idade escolar }\end{array}$ & 25 a 27 \\
\hline Sergipe (SE) & Não há descrição clara do tema no documento analisado & - \\
\hline \multicolumn{3}{|l|}{ Região Norte } \\
\hline Acre (AC) & Documento não encontrado & - \\
\hline Amapá (AP) & Não há descrição clara do tema no documento analisado & - \\
\hline Amazonas (AM) & $\begin{array}{l}\text { Situação econômica, infraestrutura, taxa de alfabetização, taxa de ocupação das pessoas de dez anos ou } \\
\text { mais de idade, população economicamente ativa e IDH }\end{array}$ & 28 a 33 \\
\hline Pará (PA) & $\begin{array}{l}\text { Aspectos socioeconômicos das } 12 \text { regiões de saúde e dados gerais referentes a saneamento básico por } \\
\text { região de saúde }\end{array}$ & 34 a 59 \\
\hline Roraima (RR) & Documento não encontrado & - \\
\hline Rondônia (RO) & Documento não encontrado & - \\
\hline Tocantins (TO) & Não há descrição clara do tema no documento analisado & - \\
\hline
\end{tabular}


Continuação - Quadro 1

\begin{tabular}{|c|c|c|}
\hline Unidade federativa & Conteúdos abordados & Páginas \\
\hline \multicolumn{3}{|l|}{ Região Sudeste } \\
\hline Espírito Santo (ES) & Aspectos sanitários, desenvolvimento socioeconômico, emprego, renda e escolaridade & 52 a 57 \\
\hline Minas Gerais (MG) & Não há descrição clara do tema no documento analisado & \\
\hline Rio de Janeiro (RJ) & $\begin{array}{l}\text { Aspectos socioeconômicos, condições de vida, trabalho e ambiente, vigilância em saúde/componente } \\
\text { ambiental, hábitos e estilos de vida }\end{array}$ & 45 a 59 \\
\hline São Paulo (SP) & Não há descrição clara do tema no documento analisado & - \\
\hline \multicolumn{3}{|l|}{ Região Sul } \\
\hline Paraná (PR) & Não há descrição clara do tema no documento analisado & - \\
\hline Rio Grande do Sul (RS) & Não há descrição clara do tema no documento analisado & - \\
\hline Santa Catarina (SC) & Aspectos socioeconômicos, ambiente e saúde, educação, promoção da saúde & $\begin{array}{c}148 \text { a } \\
162\end{array}$ \\
\hline
\end{tabular}

Fonte: Os autores.

No Plano Nacional de Saúde, o conteúdo está contemplado no tópico 1 do capítulo 1 (análise situacional), abordando de forma descritiva, em nove páginas, temas como: aspectos socioeconômicos (produto interno bruto per capita, taxa de analfabetismo, taxa de desemprego, taxa de trabalho infantil, escolaridade, razão de renda e proporção de pobres), condições de vida, trabalho e ambiente (saneamento básico, abastecimento de água, poluição atmosférica, agrotóxicos e desastres), hábitos e estilos de vida (desnutrição infantil, excesso de peso e obesidade, hábitos alimentares, obesidade, tabagismo, inatividade física e consumo excessivo de bebidas alcoólicas), complexo produtivo e de ciência, tecnologia e inovação em saúde (produção e inovação, pesquisa e desenvolvimento e regulação em saúde) (Brasil, 2011).

Nos planos estaduais de saúde dos 24 documentos recuperados e analisados, 12 não apresentavam a discussão referente ao tema dos determinantes e condicionantes de saúde. Seguem os resultados sistematizados pelas cinco regiões que dividem o território brasileiro.

\section{Região Centro-Oeste}

No Plano Estadual de Saúde do Distrito Federal, não há descrição clara do tema de determinantes e condicionantes de saúde. No capítulo da análise situacional, são abordados temas como: características demográficas do DF e do entorno, características socioeconômicas e características geográficas (Distrito Federal, 2012). O conceito aparece apenas nas diretrizes, objetivos e meta, na diretriz 1.6 (atuar nos fatores desencadeantes, determinantes e condicionantes de doenças e agravos com a finalidade de conhecer, detectar, prevenir, controlar, reduzir e eliminar riscos e danos à saúde individual ou coletiva, como acidentes e agravos relacionados ao trabalho), mas sem uma definição conceitual. O documento informa que buscou contemplar as propostas da $8^{a}$ Conferência Distrital de Saúde, realizada em 2011. 
O estado de Goiás apresenta, no capítulo de análise situacional, um item sobre determinantes e condicionantes de saúde, abordando os seguintes temas: aspectos socioeconômicos, condições de vida, trabalho e ambiente, complexo produtivo e de ciência, tecnologia e inovação em saúde. Nos aspectos socioeconômicos, são abordados dados de produção, principalmente o PIB para 2009, e é apresentado o IDH de 2007 no referido estado. Cabe ressaltar que no item condições de vida, trabalho e ambiente são explorados brevemente a questão das migrações do campo (que faz parte da análise demográfica), o abastecimento de água, o esgoto sanitário e a coleta de lixo. A apresentação do documento informa que na metodologia de construção buscou-se "compatibilizá-lo com o Plano de Governo, o Plano Plurianual (PPA) e o Plano de Ação Integrada de Desenvolvimento (PAI), na perspectiva de integração entre o planejamento e orçamento estadual e com as recomendações

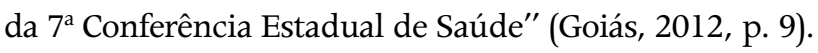

No Plano Estadual de Saúde do Mato Grosso do Sul, o tema é abordado descritivamente, no capítulo de análise situacional, posterior aos itens de condições de saúde da população e perfil epidemiológico. Há a apresentação descritiva do IDH, da taxa de urbanização, da densidade demográfica e da população em idade ativa e economicamente ativa 2006-2009, além de outros indicadores macroeconômicos, sem uma articulação entre as informações. A apresentação informa que o plano estadual de saúde foi elaborado em sintonia com os objetivos, diretrizes e ações estabelecidas pelo Ministério da Saúde para o período e com as deliberações das conferências municipais de saúde realizadas nos 78 municípios que compõem o estado (Mato Grosso do Sul, 2011).

O estado do Mato Grosso aborda o tema no tópico condições de saúde, localizado no capítulo de análise situacional, o qual abrange os seguintes itens: demografia, expectativa de vida ao nascer, índice de envelhecimento, razão de dependência, urbanização e IDH. O documento informa que utiliza como base as deliberações da Conferência Estadual de Saúde daquele estado. Nesse plano consta, na metodologia, uma abordagem conceitual para a construção do documento, a qual utilizou "a teoria Matusiana de Carlos Mattus, que entende o processo de planejamento como um 'cálculo que precede e preside a ação' (...) a construção do Plano se deu em quatro momentos. O momento explicativo, normativo, estratégico e o técnico-operacional" (Mato Grosso, 2013, p. 19).

Na região Centro-Oeste, os quatro estados informam ter utilizado como base as diretrizes das conferências estaduais de saúde. Destes, três abordam descritivamente a temática de determinantes e condicionantes de saúde, sendo que somente um preocupou-se em aprofundar conceitualmente o tema.

\section{Região Nordeste}

O Plano Estadual de Saúde de Alagoas, no capítulo de análise de situação de saúde, aborda a questão dos condicionantes e determinantes de saúde. No subtítulo 
de condicionantes e determinantes de saúde, realiza-se uma discussão teórica e são citadas a legislação do SUS e as discussões da Comissão Nacional sobre Determinantes Sociais da Saúde. Pontuam-se as principais causas de mortalidade e internação no estado, porém não se apresenta definição do quadro conceitual ou modelo utilizado de determinantes e condicionantes de saúde.

Na página 21, consta uma tabela comparativa entre o estado de Alagoas, a região Nordeste e o Brasil, apresentando os seguintes indicadores demográficos e socioeconômicos: grau de urbanização, razão de dependência (população da faixa etária economicamente ativa dependente $<15$ anos e $\geq 60$ anos em 2008), taxa de analfabetismo, PIB per capita (R\$) em 2008, proporção de pobres (\% da população com renda familiar per capita de até meio salário mínimo em 2008), taxa de desemprego, taxa de trabalho infantil, taxa de fecundidade total em 2010 e, por fim, taxa bruta padronizada de natalidade em 2007. Os dados são apresentados sem análises ou adensamentos. Não consta descrição da concepção teórica que norteou o plano. Destaca-se a parceria com a Universidade de Alagoas na elaboração do documento (Alagoas, 2012).

O Plano de Saúde da Bahia utilizou como referência o Programa Bahia Saudável, um programa setorial da saúde que seguiu o Plano Plurianual da Bahia 2012-2015, além do Projeto de Governo 2011-2014, do Plano Plurianual (PPA) e da Lei Orçamentária Anual (LOA) de 2012. O tema dos condicionantes e determinantes de saúde não é abordado de forma clara no documento analisado. No bloco da vigilância em saúde, a temática é citada nos conceitos de vigilância epidemiológica e ambiental. No capítulo VII, referente ao monitoramento e à avaliação, lê-se que o plano "foi elaborado mediante uma análise situacional da população, determinantes e condicionantes da saúde" (Bahia, 2012, p. 147); entretanto, essa análise não é apresentada no documento.

O Plano Estadual de Saúde do Ceará, em sua apresentação, destaca a compatibilização entre os instrumentos do planejamento e as recomendações da $6^{\text {a }}$ Conferência Estadual de Saúde de 2011 (Ceará, 2013). No documento, o tema de determinantes e condicionantes de saúde é abordado descritivamente, com os seguintes tópicos: aspectos econômicos, dados demográficos (distribuição da população, taxa de urbanização, densidade demográfica, crescimento e fecundidade - taxa média geométrica de crescimento populacional e taxa de fecundidade), indicadores sociais (indicadores de desenvolvimento: Índice de Desenvolvimento Humano Municipal [IDH-M], Índice de Desenvolvimento Municipal [IDM], Índice de Desenvolvimento Social de Ofertas [IDS-O] e Índice de Desenvolvimento Social de Resultado [IDS-R]), renda (renda média domiciliar per capita, renda domiciliar per capita mediana, rendimento domiciliar per capita).

Além de informações sobre saúde (cobertura populacional por ações de saúde, taxa de mortalidade infantil, expectativa de vida ao nascer), educação (número de anos de estudos, taxa de analfabetismo, percentual 
da população com ensino superior, taxa bruta de frequência ao ensino superior), habitação (número de domicílios e média de moradores, condição dos domicílios segundo situação de ocupação, acesso a bens de consumos - carro, televisão e telefone), saneamento (taxa de cobertura urbana de abastecimento de água e de esgoto sanitário, percentual de domicílios com banheiro e água encanada), coleta de lixo, atividades econômicas (agropecuária, produção agrícola, efetivo de animais) e, por fim, indicadores econômicos (PIB, PIB per capita).

No Plano Estadual de Saúde do Maranhão, o tema dos condicionantes e determinantes de saúde é apresentado em um capítulo, com sete páginas destinadas à vigilância em saúde (Maranhão, 2012). O documento aborda os seguintes tópicos: demografia (taxa média de crescimento geométrico da população, migrações, proporção de homens e mulheres, população por domicílio, cor ou raça), IDH, educação (Índice de Desenvolvimento da Educação Básica - Ideb), trabalho e renda (índice de Gini), saneamento básico (acesso à água potável, acesso aos serviços de coleta e tratamento de esgoto) e vigilância sanitária. Na introdução, consta que o plano foi elaborado com o Conselho Estadual de Saúde e buscou uma conexão das metas quadrienais do PES com o Plano Plurianual (PPA 2012-2015) maranhense. Na construção do referido plano, a Escola Técnica do SUS do Maranhão é citada como colaboradora para a sua realização.

O Plano Estadual de Saúde da Paraíba embasou-se nos relatórios finais das conferências municipais e estadual de saúde, nas diretrizes e metas do PPA e nos Objetivos de Desenvolvimento do Milênio. Segundo os autores, o documento técnico foi elaborado em um processo de participação coletiva com representantes do Conselho Estadual de Saúde e de outros atores sociais. Nesse plano, verificamos que não há referência ao tema dos determinantes e condicionantes de saúde (Paraíba, 2012). Ressalta-se que esse plano estadual de saúde foi resgatado na página do SARGSUS.

No Plano Estadual de Saúde de Pernambuco, não há referência ao tema dos determinantes e condicionantes (Pernambuco, 2012). No entanto, no capítulo 2, que trata da análise situacional, há um item que aborda descritivamente o perfil socioeconômico do estado, apresentando: IDH, educação (taxa de analfabetismo, número de analfabetos funcionais, população maior de 15 anos com menos de um ano de estudo, percentual da população com um a três anos de estudo, proporção da população com mais de oito anos de estudo), renda (renda média domiciliar per capita, proporção de pobres, proporção de pessoas que vivem com rendimento familiar de até meio salário mínimo per capita, percentual da população coberta por planos e seguros de assistência suplementar à saúde) e situação dos domicílios particulares permanentes (sexo da pessoa de referência da família, condição de atividade da pessoa de referência da família, disponibilidade de serviços, existência 
de alguns bens duráveis - com dados da Pesquisa Nacional por Amostra de Domicílios, 2009). Informa-se que a elaboração do documento conectou as metas quadrienais do PES, o Plano Plurianual e outros documentos normativos do estado e teve a participação do Conselho Estadual de Saúde e da sociedade civil organizada.

O estado do Piauí, na elaboração do seu Plano Estadual de Saúde, baseou-se nas diretrizes técnicas e normativas do Ministério da Saúde, principalmente no Plano Nacional de Saúde para o período 2012-2015, além de considerar as demandas e necessidades expressas na VI Conferência Estadual de Saúde e no Plano Plurianual do referido período (Piauí, 2013). Não há a abordagem do tema dos determinantes e condicionantes no PES. No entanto, no capítulo de análise situacional do estado, há um quadro que discrimina os seguintes índices demográficos: analfabetismo em maiores de 15 anos, taxa de crescimento populacional, proporção da população acima de 15 anos com mais de oito anos de estudo, esperança de vida ao nascer, fecundidade, proporção de idosos na população, razão de sexos, grau de urbanização, natalidade, mortalidade, mortalidade infantil, taxa de trabalho infantil e proporção de domicílios particulares por tipo de saneamento.

O Plano Estadual de Saúde do Rio Grande do Norte baseou-se no Plano Plurianual, no Relatório da $7^{\text {a }}$ Conferência Estadual de Saúde e nos documentos normativos do Ministério da Saúde e da Secretaria de Saúde do referido estado (Rio Grande do Norte, 2013). Os condicionantes e determinantes de saúde são denominados 'condicionantes e determinantes socioeconômicos' e apresentados, sucintamente, nos seguintes índices: IDH, taxa de urbanização, indicadores de saneamento básico (acesso aos sistemas de água potável, esgotamento sanitário e coleta de resíduos sólidos), Indicador Social de Desenvolvimento dos Municípios (ISDM, que abrange habitação, renda, trabalho, saúde e educação e ainda 28 variantes desses componentes), renda familiar per capita abaixo da linha da pobreza, índice de mortalidade infantil, índice de doenças evitáveis em crianças menores de cinco anos, Índice de Desenvolvimento da Educação Básica (IDEB) e proporção da população em idade escolar.

No Plano Estadual de Saúde de Sergipe, na introdução consta a descrição da participação dos conselheiros estaduais de saúde na elaboração do referido documento. O tema dos condicionantes e determinantes não é abordado diretamente. Na análise situacional, apresenta-se uma tabela com diversos indicadores sociais, comparando-se o estado de Sergipe com a região Nordeste e o Brasil. O termo determinantes e condicionantes aparece somente para conceituar as divisões da vigilância em saúde desse estado (Sergipe, 2012).

Ressalta-se que quatro dos nove planos estaduais de saúde da região Nordeste não abordam de forma clara e consistente o tema estudado. 


\section{Região Norte}

No Plano Estadual de Saúde do Amapá, aponta-se que o PES incorporou as propostas da VI Conferência Estadual de Saúde do Amapá e as prioridades nacionais definidas pelo Ministério da Saúde no Plano Nacional de Saúde e no Pacto pela Saúde, entre outros documentos normativos daquele estado (Amapá, 2011). Não há a abordagem do tema dos determinantes e condicionantes de saúde no plano.

O Plano Estadual de Saúde do Amazonas segue o referencial do planejamento do SUS. Na análise situacional, o tema dos condicionantes e determinantes de saúde é abordado descritivamente por meio dos seguintes itens: situação econômica (indicadores econômicos tais como PIB, produtos agrícolas, produtos minerais, produtos da pecuária, extrativismo vegetal, indústria e arrecadação de Imposto sobre Operações Relativas à Circulação de Mercadorias e Prestação de Serviços de Transporte Interestadual e Intermunicipal e de Comunicação ICMS), infraestrutura (coleta de lixo, distribuição de energia elétrica, consumo de energia), taxa de alfabetização, taxa de ocupação das pessoas de dez anos ou mais de idade, população economicamente ativa e IDH (Amazonas, 2012).

Já o Plano Estadual de Saúde do Pará contemplou o tema de determinantes e condicionantes de saúde no item "determinantes sociais de saúde". Tal tópico aborda os aspectos socioeconômicos das 12 regiões de saúde e dados gerais referentes a saneamento básico por região de saúde. No início da página 34, são citados a lei n. 8.080/1990, que institui o SUS, e o conceito de determinantes sociais de saúde. Destaca-se que o governo do Estado do Pará traçou cinco grandes diretrizes, entendendo que o tema dos determinantes sociais de saúde transcende a área da saúde. O documento afirma seguir o referencial do planejamento normativo do Ministério da Saúde (Pará, 2012).

O estado do Tocantins, no Plano Estadual de Saúde, utilizou como proposta metodológica o planejamento estratégico, assim como as diretrizes do Sistema de Planejamento do SUS (PlanejaSUS) e normatizações estaduais. Apesar de ter um subcapítulo na parte I de análise da situação de saúde denominado "condições de saúde da população, determinantes e condicionantes", esse tema não é claramente abordado (Tocantins, 2012).

Nessa região, três planos estaduais de saúde não foram recuperados. Destaca-se o Plano de Saúde do estado do Pará, o qual contemplou o tema de determinantes e condicionantes de saúde e traçou diretrizes a partir de uma concepção ampliada de saúde.

\section{Região Sudeste}

No Espírito Santo, o Plano Estadual de Saúde tem um tópico abordando os determinantes e condicionantes de saúde, o qual apresenta os seguintes pontos: aspectos 
sanitários (domicílios com rede geral de esgoto ou ligados à rede pluvial e consumo de agrotóxicos), desenvolvimento socioeconômico (taxa de pobreza) e emprego, renda e escolaridade (trabalho formal, população economicamente ativa/pessoas de 15 anos ou mais de idade, ocupadas ou que procuram emprego, escolaridade média dos adultos - 25 anos ou mais -, taxa de analfabetismo e analfabetismo funcional, e ocorrência de homicídios). Nos aspectos sanitários, são citados sistemas da vigilância em saúde como Vigiar, Vigiágua e Sissolo. Das 17 diretrizes do documento, a $7^{\mathrm{a}}$ aborda a implementação das ações de promoção considerando os determinantes e condicionantes da saúde (Espírito Santo, 2012).

O Plano Estadual de Saúde de Minas Gerais, que buscou contemplar as diretrizes da VII Conferência Estadual de Saúde do estado, teve na sua elaboração a participação de diversas entidades daquela unidade federativa, incluindo o Conselho Estadual de Saúde, a Escola de Saúde Pública, a Fundação Ezequiel Dias e a Fundação Hospitalar do Estado de Minas Gerais. Não há descrição clara sobre o tema dos determinantes e condicionantes de saúde no plano, embora apareça na diretriz "implementar ações de abordagem aos fatores condicionantes, determinantes, riscos e danos à saúde, inclusive ações de saneamento básico e saúde ambiental" a discussão teórica sobre o tema. “...os determinantes e os condicionantes de saúde, conceituados como as condições em que as pessoas vivem e trabalham, ou como as características dentro das quais a vida transcorre, são o foco de atuação da Vigilância" (Minas Gerais, 2013, p. 99).

Nesse plano estadual, é apresentado um fator de alocação do repasse financeiro estadual para os municípios, o qual foi construído a partir da associação de dois índices: o Índice de Necessidade em Saúde (INS), que congrega um conjunto de seis variáveis que procuram medir as condições epidemiológicas e socioeconômicas dos municípios - entre elas, mortalidade da criança, proporção de óbitos por causas mal definidas, taxa de fecundidade, taxa de alfabetização, percentual de indivíduos com renda domiciliar menor que o salário mínimo e proporção de pessoas que vivem em domicílios com coleta de lixo -, e o Índice de Porte Econômico (IPE), que corresponde ao valor per capita do ICMS de cada município, trabalhado por uma expressão logarítmica. Esse índice expressa a capacidade do município em financiar, com recursos próprios, os cuidados com a saúde de seus cidadãos.

No Plano de Saúde do estado do Rio de Janeiro, o tema foi contemplado (Rio de Janeiro, 2012). Na apresentação e introdução do documento, lê-se sobre a parceria com o Conselho de Secretários Municipais de Saúde e o Conselho Estadual de Saúde, assim como o seguimento do Plano Plurianual e das propostas anuais de diretrizes orçamentárias daquele estado. Para a produção do PES são referidas as proposições do PlanejaSUS, seguindo a estrutura adotada pelo Ministério da Saúde para a elaboração do Plano Nacional de Saúde 2012-2015. Dos três eixos que compõem o plano, um trata especificamente das questões de determinantes e condicionantes de saúde, abordando os seguin- 
tes itens: aspectos socioeconômicos, condições de vida, trabalho e ambiente, vigilância em saúde e hábitos e estilos de vida.

Em São Paulo, a elaboração e a execução do PES ocorreram em sintonia com o Pacto pela Saúde no referido estado, contando com a participação da Comissão Intergestores Bipartite e do Conselho Estadual de Saúde. O plano desse estado (São Paulo, 2012) seguiu o referencial do PlanejaSUS, tratando-se de um instrumento técnico-político que teve como subsídio as proposições das conferências de saúde nacional e do estado de São Paulo de 2011. Apesar de citar o artigo $5^{\circ}$ da portaria n. 3.332/2006 (Brasil, 2006), que define os eixos orientadores para a análise situacional e para a formulação dos objetivos, diretrizes e metas dos planos de saúde, dentre eles o tópico dos determinantes e condicionantes de saúde, esse tema não é abordado no documento.

Dois dos quatro planos estaduais de saúde da região Sudeste não abordaram claramente o tema estudado. No entanto, nos documentos analisados, a discussão sobre determinantes e condicionantes aparece sublinhada como importante na construção desses documentos técnicos.

\section{Região Sul}

O Plano Estadual de Saúde do Paraná foi realizado em parceria com o Conselho Estadual de Saúde daquele estado. No documento, não é apresentado diretamente o tema dos determinantes e condicionantes de saúde (Paraná, 2013). Entretanto, no capítulo 2, que trata da análise da situação de saúde, é descrito o perfil socioeconômico e demográfico do estado, em que os seguintes itens são abordados: taxa de urbanização, estrutura etária (taxa média geométrica anual), razão de sexo, taxa de fecundidade, alfabetização e nível de instrução (taxa de alfabetização, pessoas de dez anos ou mais de idade segundo o nível de instrução), saneamento (taxa de domicílios particulares permanentes sem banheiro ou sanitário), rendimento domiciliar e salários dos ocupados formais (taxa de pobreza domiciliar/proporção ou taxa de domicílios particulares permanentes com rendimento mensal nominal per capita de até $1 / 4$ de salário mínimo, salários médios ou rendimento mensal médio nominal dos empregados formais) e PIB.

No texto, lê-se na diretriz 4, que trata do fortalecimento da rede de atenção à saúde para pessoas com sofrimento ou transtorno mental e com necessidades decorrentes do uso do crack, álcool e outras drogas, o seguinte excerto: "é um desafio construir um sistema de saúde capaz de enfrentar os determinantes e condicionantes da saúde, pois requer a estruturação de uma Rede de Atenção em Saúde organizada a partir da Atenção Primária em Saúde" (Paraná, 2013, p. 179). Contudo, não está relatada a abordagem de como foi proposto trabalhar o referido tema.

No estado do Rio Grande do Sul, o tema dos determinantes e condicionantes de saúde não está descrito diretamente no Plano Estadual de Saúde. Na intro- 
dução do documento técnico, lê-se que ele foi compartilhado e amplamente discutido com o Conselho Estadual de Saúde, além de contar com a participação do Conselho das Secretarias Municipais de Saúde do Rio Grande do Sul (Cosems/RS). O PES ainda "teve como orientador o Decreto Presidencial n. 7.508/2011 e como subsídios o desenho do Mapa Estratégico do Governo, o Plano Plurianual Participativo 2012-2015 e a definição do novo desenho das Regiões de Saúde" (Rio Grande do Sul, 2013, p. 14).

Nesse documento, são apresentadas medidas como o Índice de Desenvolvimento Socioeconômico (Idese), elaborado pela Fundação de Economia e Estatística (FEE) desse estado, que considera os seguintes blocos de indicadores: domicílio, saneamento, educação e saúde e renda; Índice de Vulnerabilidade Social (IVS), que classifica os municípios do estado em ordem crescente de vulnerabilidade; e o Índice Regional (IR), utilizado para estabelecer valores diferenciados para o pagamento de cirurgias eletivas nas diferentes regiões de saúde do estado. São abordados também o IDH, o PIB per capita, dados demográficos (sexo, faixa etária, percentual da população jovem, trabalhadora e idosa, razão de dependência, coeficiente de natalidade, expectativa de vida/esperança de vida, cor/raça) e dados sobre grupos específicos (população negra, população indígena e população privada de liberdade).

A menção sobre determinantes e condicionantes de saúde aparece no título "determinantes ambientais de morbimortalidade", abordando os fatores de risco e determinantes do processo saúde na perspectiva da vigilância em saúde. São discutidos aspectos teóricos do modelo de produção como a exposição humana aos agrotóxicos, a vigilância da qualidade da água para consumo humano, a vigilância de populações expostas aos poluentes atmosféricos (Vigiar), a vigilância de eventos ambientais adversos à saúde e as intoxicações.

No Plano Estadual de Saúde de Santa Catarina, há um capítulo específico que aborda o tema avaliado, denominado "eixo dos determinantes e condicionantes de saúde" (Santa Catarina, 2011). São abordados quatro itens: aspectos socioeconômicos (PIB per capita, razão de renda, proporção de pobres e taxa de desemprego, taxa de trabalho infantil), ambiente e saúde (saneamento e poluição ambiental: percentual de famílias abastecidas por rede geral de abastecimento de água, percentual de famílias atendidas direta ou indiretamente por serviço regular de coleta de lixo, percentual de famílias com cobertura de esgotamento sanitário, percentual de famílias com cobertura de saneamento básico, percentual de amostras fora do padrão para coliformes totais), educação (porcentagem de alfabetizados na população com idade de 15 anos ou mais - taxa de analfabetismo, escolaridade/anos de estudos - e percentual de indivíduos com oito ou mais anos de escolaridade) e promoção da saúde.

Cabe ressaltar que as divisões da vigilância em saúde desse estado foram abordadas no capítulo de determinantes e condicionantes de saúde com os seguintes temas: vigilância da qualidade da água para consumo 
humano, resíduos de serviços de saúde, vigilância em saúde de populações expostas a solo contaminado (Vigisolo), vigilância em saúde ambiental relacionada à qualidade do ar (Vigiar), vigilância em saúde ambiental dos riscos decorrentes dos desastres naturais (Vigidesastres), vigilância em saúde ambiental relacionada a acidentes com produtos perigosos (Vigiapp), monitoramento ambiental do cólera e vigilância em saúde ambiental relacionada às substâncias químicas.

Os autores apontam que utilizaram como método para o desenvolvimento do trabalho o enfoque estratégico-situacional desenvolvido por Carlos Matus e que foram analisados documentos técnicos do planejamento em âmbitos nacional e estadual.

Na região Sul, apenas o estado de Santa Catarina tem um capítulo específico em seu plano estadual de saúde que aborda o tema avaliado.

\section{Pluralidade de abordagens teóricas e práticas sobre determinantes e condicionantes: perspectivas e desafios}

Observou-se que, embora os determinantes sociais de saúde sejam expressos de formas diversas nos textos dos planos de saúde, este é um tema pouco definido, realidade constatada nos documentos analisados para o período de 2012 a 2015.

Em nenhum dos planos de saúde analisados se encontra uma discussão clara sobre o modelo de determinantes e condicionantes de saúde utilizado para a realização das análises. Essa constatação é importante, uma vez que a definição do modelo adotado é uma etapa importante para a construção das análises, pois indica quais critérios serão adotados e priorizados e como eles se relacionam entre si.

De forma geral, verificou-se que, na construção tanto do Plano Nacional quanto dos planos estaduais de saúde, os indicadores utilizados foram apresentados de forma não sistematizada e sem clareza conceitual. Percebe-se, ainda, que são apresentados indicadores demográficos, socioeconômicos, de mortalidade (principalmente taxa de mortalidade infantil e razão de mortalidade materna), de morbidade e fatores de risco. No entanto, não é apontado como esses dados se articulam para compor a análise dos determinantes e condicionantes de saúde. Além disso, aparece de forma desarticulada a apresentação do assunto a partir da organização da vigilância em saúde (sanitária, epidemiológica, ambiental e do trabalhador).

Contemplar condicionantes e determinantes de saúde na elaboração dos planos é evitar que iniquidades em saúde se solidifiquem nos sistemas de saúde e políticas públicas. Nesse sentido, alguns índices têm sido construídos no sentido de produzir estratégias de financiamento para diminuir essas desigualdades em saúde - como exemplo, o Índice de Vulnerabilidade Social, 
adotado pelo estado do Rio Grande do Sul para alocação de recursos financeiros (Drachler et al., 2014).

É importante ressaltar também que a maior parte dos planos de saúde analisados utiliza dados desagregados descritivos sobre itens que compõem a abordagem dos determinantes e condicionantes, porém sem uma análise ou um adensamento. Poucos estados usam índices compostos para sumarizar diferentes temas, buscando fazer uma relação entre eles. Nesse sentido, salienta-se que os índices mais utilizados foram o IDH e o PIB.

As pesquisas populacionais e demográficas mais utilizadas nos documentos foram as do Instituto Brasileiro de Geografia e Estatística (IBGE) - censo demográfico, contagem da população, estimativas populacionais, Pesquisa Nacional por Amostra de Domicílios (PNAD), Pesquisa de Orçamentos Familiares (POF) - e pesquisas do Ministério da Saúde - vigilância de fatores de risco e proteção para doenças crônicas por inquérito telefônico (Vigitel) e Pesquisa Nacional sobre Demografia e Saúde (PNDS).

Ressalta-se que o modelo teórico a ser escolhido deve ser capaz de retratar o cenário em análise, considerando as medidas sumárias (indicadores ou índices) possíveis de serem utilizadas, bem como a capacidade técnica, política e institucional. Portanto, aponta-se a urgência de os planejadores se debruçarem sobre quadros conceituais para a abordagem do tema com clareza e consistência e assim utilizá-los para o planejamento em saúde. A discussão de determinantes e condicionantes nos planos de saúde brasileiros precisa avançar no sentido de qualificar e nortear as ações e programas a serem executados nos territórios. Dessa forma, é importante que se façam análises e estudos sobre os modelos de determinantes e condicionantes de saúde disponíveis, a fim de paulatinamente inseri-los, de forma consistente, na discussão das políticas públicas de saúde.

Nos planos estaduais de saúde analisados, percebeu-se que a maioria dos documentos utilizou apenas o referencial do planejamento em saúde numa perspectiva normativa, conforme proposto em documentos técnicos do $\mathrm{Mi}$ nistério da Saúde. Observou-se um esforço no sentido de qualificar a parte de análise situacional.

A relação com universidades e escolas de saúde pública foi outro ponto relevante apontado na elaboração de alguns documentos analisados, pois pode ser considerada positiva para qualificar a gestão, engajar as esferas governamentais e integrar ensino/formação/serviço na área da saúde.

Outro destaque presente nos planos estaduais de saúde, também presente no Plano Nacional de Saúde, foi a utilização das deliberações das conferências de saúde, guardando coerência com o que é preconizado no processo de planejamento. Essa informação remeteu a um esforço de aproximação entre o controle social e a gestão da saúde, premissa básica da construção do SUS. 
Para o planejamento, pensar no conjunto de determinações sociais que afetam a saúde das populações é primordial para sua prática. O combate às desigualdades sociais por meio de políticas públicas intersetoriais precisa estar presente na agenda política dos gestores, ou seja, os interesses políticos e econômicos devem estar alinhados aos técnicos e sociais. Igualmente, os planejadores em saúde precisam ocupar-se mais de questões metodológicas que avaliem como os determinantes sociais em saúde afetam o processo saúde-doença e como a presença ou ausência de políticas públicas pode contribuir para esse cenário.

Ressalta-se que, na área do planejamento em saúde, também se faz necessário considerar o paradigma histórico-estrutural além do conceito de causa e a visão biologicista da epidemiologia tradicional (Nogueira, 2009). Essas questões devem estar contempladas nos planos de saúde brasileiros, desde o Plano Nacional (que induz os planos das outras esferas públicas), passando pelos planos estaduais, até os planos municipais de saúde.

$\mathrm{Na}$ análise realizada, priorizou-se a parte inicial do Plano Nacional e dos planos estaduais de saúde - a análise situacional, entendendo-se que na apresentação do quadro conceitual é que precisa estar definida pragmaticamente a abordagem dos determinantes e condicionantes de saúde. Contudo, na segunda parte dos planos, denominada diretrizes, objetivos e metas, encontrou-se em alguns documentos a utilização do conceito analisado sem que ele tivesse sido discutido na definição conceitual.

Em alguns planos estaduais de saúde, o tema analisado apareceu posteriormente à avaliação da morbidade e mortalidade, ou antes da análise demográfica. Porém, reitera-se que condicionantes e determinantes de saúde devem estar no 'entre', após a análise demográfica e antes da morbidade e mortalidade, pois esses aspectos relacionam-se ao modo como as pessoas vivem, produzem e são produzidas, adoecem e morrem, nos diferentes territórios e contextos sociais. Em uma linha contínua, os determinantes e condicionantes de saúde são o início de uma cadeia de produção de estados de saúde, e no intermédio estão as políticas sociais e as políticas públicas de saúde.

Caso não haja um efetivo investimento em saúde, o fim da cadeia podem ser as iniquidades em saúde (Buss e Pellegrini Filho, 2007). Por essa razão, alguns indicadores de determinantes e condicionantes de saúde são correlacionados com indicadores que medem iniquidades em saúde.

\section{Considerações finais}

A apresentação dos determinantes e condicionantes de saúde foi abordada de forma breve em alguns dos planos de saúde analisados para o período de 2012 a 2015. Verificou-se que foram utilizados dados de pesquisas demográficas, estudos de prevalência e dados secundários de bases de dados federais para caracterizar diversos tópicos que compõem o tema, mas sem uma clara definição conceitual. 
Ao se entender que os planos de saúde são instrumentos oficiais e obrigatórios que expressam a forma como os gestores em saúde concebem, planejam e priorizam ações e programas de saúde para a construção de políticas públicas, o estudo aqui apresentado apontou a necessidade de maior atenção e clareza quanto à apropriação pelos gestores e planejadores em saúde do conceito de determinantes em saúde. Faz-se necessária a revisão dos modelos de determinantes e condicionantes de saúde, os quais irão sustentar a elaboração de documentos técnicos que fomentarão ações e programas em saúde nos diferentes municípios, estados e na União.

Por fim, ressalta-se que a análise dos determinantes e condicionantes de saúde, pensados com base em modelos sobre o tema, pode ser uma ferramenta potente para qualificar a análise situacional e ao mesmo tempo servir como meio para a área do planejamento, monitoramento e avaliação, no sentido de que se possa construir políticas públicas que contribuam para que a gestão em saúde impacte positivamente as condições de vida das populações.

\section{Colaboradores}

Os autores trabalharam conjuntamente em todas as etapas do manuscrito.

Resumen El objetivo de la investigación fue describir cómo el tema de los determinantes y condicionantes de salud fue contemplado en el plan nacional y en los planes estaduales de salud preparados para el período de 2012 a 2015. Se trató de una investigación cualitativa, de tipo análisis documental, con foco en el tema de los determinantes y condicionantes de salud. Los referidos documentos se buscaron en la página del Ministerio de Salud y en las secretarías estaduales de salud de las 27 unidades federativas brasileñas. La búsqueda también se realizó en el Sistema de Apoyo a la Construcción del Informe de Gestión, en el website Google y con el envío de correos electrónicos. Se observó que el tema de los determinantes y condicionantes de salud está poco presente en los planes de salud brasileños. La mayoría de los documentos utilizó la referencia de la planificación en salud en perspectiva normativa, según propuesto en documentos técnicos del Ministerio de Salud. La discusión sobre modelos de determinantes y condicionantes de salud no estaba presente en los documentos analizados, y hubo una descripción mixta del tema por medio de datos desagregados e índices compuestos. El estudio indicó la necesidad de mayor atención y revisión de los modelos de determinantes y condicionantes de salud empleados en la construcción de estos documentos técnicos.

Palabras clave determinantes sociales de la salud; planes gubernamentales de salud; planeamiento en salud; Sistema Único de Salud. 


\section{Notas}

${ }^{1}$ Secretaria Estadual de Saúde do Rio Grande do Sul, Porto Alegre, Rio Grande do Sul, Brasil. <andrequevedo_sls@hotmail.com>

Correspondência: Rua Demétrio Ribeiro, 988, apto. 204, Centro, CEP 90010-313, Porto Alegre, Rio Grande do Sul, Brasil.

${ }^{2}$ Secretaria Estadual de Saúde do Rio Grande do Sul, Porto Alegre, Rio Grande do Sul, Brasil. $<$ carmenbagatini@gmail.com>

${ }^{3}$ Pontifícia Universidade Católica do Rio Grande do Sul, Porto Alegre, Rio Grande do Sul, Brasil. $<$ maria.bellini@pucrs.br>

${ }^{4}$ Secretaria Estadual de Saúde do Rio Grande do Sul, Porto Alegre, Rio Grande do Sul, Brasil. $<$ rebel-machado@saude.rs.gov.br>

${ }^{5}$ Secretaria Estadual de Saúde do Rio Grande do Sul, Porto Alegre, Rio Grande do Sul, Brasil. <camilaguaranha@gmail.com>

\section{Referências}

ALAGOAS. Secretaria de Estado da Saúde. Superintendência de Gestão e Participação Social. Plano Estadual de Saúde do Quadriênio 2012-2015 (PES 2012-2015). Maceió: Sesau/ $\mathrm{AL}$, 2012. Disponível em: <http://www. saude.al.gov.br/wp-content/uploads/2016/03/ PES_2012_2015.pdf > . Acesso em: 7 abr. 2015.

AMAPÁ. Governo do Estado do Amapá. Secretaria Estadual da Saúde. Plano Estadual de Saúde 2012-2015. Macapá, 2011.

AMAZONAS. Secretaria de Estado de Saúde do Amazonas (Susam). Planos e Relatórios. Plano Estadual de Saúde-PES 2012-2015. Manaus, 2012. Disponível em: <http://www.saude.am.gov. br/docs/pes/pes_2012-2015_revisao_01.pdf>. Acesso em: 9 abr. 2015.

BAHIA. Secretaria da Saúde. Gestão da Saúde. Plano Estadual de Saúde 2012-2015. Revista Baiana de Saúde Pública, Salvador, v. 36, supl. 1, dez. 2012. Disponível em: <http://www. saude.ba.gov.br/images/stories/profissional_e_gestor/gestao_da_saude/RBSP_Suplemento_2012_completo_02mar2013.pdf > . Acesso em: 7 abr. 2015.
BARDIN, Laurence. Análise de conteúdo. Tradução de Luís Antero Reto e Augusto Pinheiro. Lisboa: Edições 70, 2010.

BRASIL. Ministério da Saúde. Gabinete do Ministro. Portaria n. 3.332, de 28 de dezembro de 2006. (Revogada pela PRT GM/MS n. 2.135, de 25.09.2013.) Aprova orientações gerais relativas aos instrumentos do Sistema de Planejamento do SUS. Disponível em: $<$ http://bvsms.saude.gov.br/bvs/saudelegis/gm/2006/prt3332_28_12_2006.html>. Acesso em: 21 mar. 2015.

BRASIL. Ministério da Saúde. Secretaria Executiva. Subsecretaria de Planejamento e Orçamento. Plano Nacional de Saúde PNS 2012-2015. Brasília: Ministério da Saúde, 2011. Disponível em: <http://bvsms. saude.gov.br/bvs/publicacoes/plano_nacional_saude_2012_2015.pdf $>$. Acesso em: 7 abr. 2015.

BRASIL. Ministério da Saúde. Gabinete do Ministro. Portaria n. 2.135, de 25 de setembro de 2013. Estabelece diretrizes para o processo de planejamento no âmbito do Sistema Único de Saúde 
(SUS). Disponível em: <http://bvsms.saude.gov. br/bvs/saudelegis/gm/2013/prt2135_25_09_2013. html>. Acesso em: 21 mar. 2015.

BUSS, Paulo M.; PELLEGRINI FILHO, Alberto. Iniquidades em saúde no Brasil, nossa mais grave doença: comentários sobre o documento de referência e os trabalhos da Comissão Nacional sobre Determinantes Sociais da Saúde. Cadernos de Saúde Pública, Rio de Janeiro, v. 22, n. 9, p. 2.005-2.008, 2006. Disponível em: $<$ http://www.scielo.br/pdf/csp/v22n9/26.pdf > . Acesso em: 17 abr. 2015.

BUSS, Paulo M.; PELLEGRINI FILHO, Alberto. A saúde e seus determinantes sociais. Physis: Revista de Saúde Coletiva, Rio de Janeiro, v. 17, n. 1, p. 77-93, 2007. Disponível em: <http://www.scielo.br/pdf/physis/v17nl/ v17nla06.pdf $>$. Acesso em: 23 abr. 2015.

CEARÁ. Secretaria da Saúde. Políticas de Saúde. Plano Estadual de Saúde 2012-2015. Fortaleza, 2013.

COMISSÃO NACIONAL SOBRE DETERMINANTES SOCIAIS DA SAÚDE. Rumo a um modelo conceitual para análise e ação sobre os determinantes sociais de saúde. Rio de Janeiro: CSDH, 2005. Disponível em: <http://www. bvsde.paho.org/bvsacd/cd57/comissao.pdf $>$. Acesso em: 21 ago. 2015.

DISTRITO FEDERAL. Secretaria de Estado de Saúde. Plano Distrital de Saúde 2012 a 2015. Brasília, 2012. Disponível em: $<$ http://www.saude.df.gov.br/images/LAI/ planodesaude_2012_2015.pdf $>$. Acesso em: 7 abr. 2015.

DRACHLER, Maria L. et al. Desenvolvimento e validação de um índice de vulnerabilidade social aplicado a políticas públicas do SUS. Ciência \& Saúde Coletiva, Rio de Janeiro, v. 19, n. 9, p. 3.849-3.858, 2014. Disponível em: <http://www.scielo.br/pdf/csc/v19n9/ 1413-8123-csc-19-09-3849.pdf $>$. Acesso em: 7 abr. 2015.

ESPÍRITO SANTO. Portal do Governo do Espírito Santo. Secretaria da Saúde. Plano Estadual de
Saúde 2012-2015. Vitória, 2012. Disponível em: $<$ http://docplayer.com.br/2929925-Estado-do-espirito-santo-secretaria-de-estado-da-saude-plano-estadual-de-saude-2012-2015.html>. Acesso em: 7 abr. 2015.

GOIÁS. Secretaria de Estado de Saúde. Acesso à Informação. Plano Estadual de Saúde-PES 2012-2015. Goiânia, 2012. Disponível em: $<$ http://www.sgc.goias.gov.br/upload/links/ arq_944_planoestadualdesaude2012-2015.pdf > . Acesso em: 7 abr. 2015.

MARANHÃO. Governo do Estado. Secretaria de Estado da Saúde. Assessoria de Planejamento. Plano Estadual de Saúde do Maranhão 2012-2015. São Luís: Secretaria de Estado da Saúde, 2012. Disponível em: <http://www. youblisher.com/p/746409-pesma2012>. Acesso em: 7 abr. 2015.

MATO GROSSO DO SUL. Secretaria de Estado de Saúde. Planejamento. Plano Estadual de Saúde-PES 2012-2015 (revisado em 17/06/2013), Campo Grande, 2011.

MATO GROSSO. Secretaria de Estado de Saúde. Plano Estadual de Saúde Mato Grosso 20122015. Cuiabá, 2013. Disponível em: <www. saude.mt.gov.br/arquivo/4608>. Acesso em: 7 abr. 2015 .

MINAS GERAIS. Secretaria Estadual de Saúde. Sistema de Gestão do SUS. Plano Estadual de Saúde 2012/2015. Belo Horizonte, 2013. Disponível em: <http://www.saude.mg.gov.br/ images/documentos/Plano \%20estadual $\% 20$ de\%20saude_210213_BX.pdf $>$. Acesso em: 7 abr. 2015 .

MINAYO, Maria C. S. O desafio do conhecimento: pesquisa qualitativa em saúde. 14. ed. Rio de Janeiro: Hucitec, 2014.

NOGUEIRA, Roberto P. Determinantes, determinação e determinismos sociais. Saúde em Debate, Rio de Janeiro, v. 33, n. 83, p. 397-406, 2009.

PARAÍBA. Secretaria de Estado da Saúde. Plano Estadual de Saúde. Paraíba 2012-2015. João Pessoa, 2012. 
PARANÁ. Secretaria de Estado da Saúde. Conselho Estadual de Saúde. Plano Estadual de Saúde Paraná 2012-2015. Curitiba, 2013. Disponível em: <http://www.saude.pr.gov.br/ arquivos/File/plano_estadual_de_saude_versao_final.pdf $>$. Acesso em: 7 abr. 2015.

PARÁ. Secretaria de Estado e Saúde Pública. Plano Estadual de Saúde do Pará 2012-2015. Belém, 2012. Disponível em: <https://www2. mppa.mp.br/sistemas/gcsubsites/upload/37/ PES-2012-2015.pdf>. Acesso em: 10 abr. 2015.

PERNAMBUCO. Secretaria Estadual de Saúde. Plano Estadual de Saúde 2012-2015. Recife, 2012. Disponível em: <http://portal.saude. pe.gov.br/sites/portal.saude.pe.gov.br/files/ plano_estadual_de_saude_2012-2015_0.pdf > . Acesso em: 7 abr. 2015.

PIAUÍ. Secretaria de Estado da Saúde. Portal da Saúde. Plano Estadual de Saúde 2012/2015. Teresina, 2013. Disponível em: <http:// www.saude.pi.gov.br/avisos-informes $>$. Acesso em: 14 abr. 2015.

RIO DE JANEIRO. Secretaria de Saúde. Informes ao Gestor. Instrumentos de Planejamento do SUS. Plano Estadual de Saúde (PES) 20122015. Rio de Janeiro, 2012. Disponível em: <http://www.saude.rj.gov.br/comum/code/ MostrarArquivo.php?C $=\mathrm{NjMwNw} \% 2 \mathrm{C} \% 2 \mathrm{C}>$. Acesso em: 7 abr. 2015.

RIO GRANDE DO NORTE. Secretaria de Saúde Pública. Plano Estadual de Saúde 2012-2015. Natal, 2013. Disponível em: <http://adcon.rn.gov.br/ACERVO/sesap/DOC/ DOC000000000004541.PDF\#sthash.H2pFXie9. dpuf>. Acesso em: 7 abr. 2015.

RIO GRANDE DO SUL. Secretaria da Saúde. Plano Estadual de Saúde 2012/2015. Porto Alegre, 2013.
Disponível em: <http://www.saude.rs.gov.br/ upload/arquivos/201703/28101537-pes-2012-2015. pdf>. Acesso em: 7 abr. 2015.

SANTA CATARINA. Secretaria de Estado da Saúde. Planejamento em Saúde. Instrumentos de Gestão Estadual. Plano Estadual de Saúde 2012-2015. Florianópolis, 2011. Disponível em: <http://portalses.saude.sc.gov.br/index. php?option $=$ com_docman\&Itemid $=251>$. Acesso em: 7 abr. 2015.

SÃO PAULO. Secretaria de Estado da Saúde. Documentos de Planejamento em Saúde. Plano Estadual de Saúde 2012-2015. São Paulo, 2012. Disponível em: <http://www.saude.sp.gov. br/resources/ses/perfil/gestor/documentos-de-planejamento-em-saude/plano-estadual-de-saude-2012-2015-sessp/pes_2012_2015.pdf>. Acesso em: 7 abr. 2015.

SERGIPE. Plano Estadual de Saúde. Quadriênio 2012-2015. Aracajú, 2012.

SISTEMA DE APOIO AO RELATÓRIO DE GESTÃO. SARGSUS. DATASUS. SGEP - Secretaria de Gestão Estratégica e Participativa. Disponível em: <http://aplicacao.saude.gov.br/ sargsus/login!carregarPagina.action $>$. Acesso em: 17 abr. 2015.

TOCANTINS. Secretaria da Saúde. Gabinete do Secretário. Diretoria Geral de Gestão e Acompanhamento Estratégico. Plano Estadual de Saúde 2012-2015. Palmas: Secretaria de Estado da Saúde, 2012. Disponível em: $<$ https://central3.to.gov.br/arquivo/241754/>. Acesso em: 9 abr. 2015.

Recebido em 24/10/2015

Aprovado em 05/12/2016 